\title{
Can phrenic stimulation protect the diaphragm from mechanical ventilation- induced damage?
}

\author{
To the Editor:
}

Mechanical ventilation is a prominent lifesaving treatment. It is, however, associated with an array of adverse effects, which include ventilator-associated pneumonias, volume-induced lung injury and, more recently identified, ventilator-induced diaphragm dysfunction (VIDD) [1-3]. VIDD combines diaphragm weakness with muscle fibre atrophy, remodelling and injury. Its mechanisms involve decreased protein synthesis, increased proteolysis, increased oxidative stress and mitochondrial dysfunction [2, 4]. Controlled mechanical ventilation appears to be the main, if not the sole, risk factor for VIDD, which in animal models is attenuated by the maintenance of respiratory efforts (assisted ventilatory modes) [2]. Although the corresponding human evidence is still lacking, this underlies the current notion that "clinicians should encourage persistent diaphragmatic activity" in patients receiving mechanical ventilation [2]. Diaphragm pacing has been proposed as a surrogate for spontaneous respiratory activity when the latter is not compatible with the condition of the patient $[2,5]$, but this approach has, seemingly, not yet been tested experimentally.

Here we report a preliminary description of putative beneficial effects of diaphragm pacing in three mechanically ventilated sheep. Three female adult sheep (41, 32 and $34 \mathrm{~kg}$ ), were anaesthetised (premedication: acepromazine $1.3 \mathrm{mg} \cdot \mathrm{kg}^{-1}$ i.m., $30 \mathrm{~min}$ before induction; induction: propofol $6 \mathrm{mg} \cdot \mathrm{kg}^{-1}$ i.v.; maintenance: continuous propofol $1-2 \mathrm{mg} \cdot \mathrm{kg}^{-1} \cdot \mathrm{h}^{-1}$, midazolam $0.3-2 \mathrm{mg} \cdot \mathrm{kg}^{-1} \cdot \mathrm{h}^{-1}$ and morphine $0.2-$ $0.3 \mathrm{mg} \cdot \mathrm{kg}^{-1} \cdot \mathrm{h}^{-1}$; no paralysing agents), tracheotomised and mechanically ventilated with a minute ventilation ensuring normocapnia (Aisys, GE Healthcare, Datex Ohmeda, Madison, WI, USA). Additional oxygen was given to maintain transcutaneous-pulsed oxygen saturation $>92 \%$. Adequate fluid and nutritional support was provided and glycaemia controlled. Body temperature, heart rate and arterial pressure were monitored. Intradiaphragmatic phrenic nerve stimulation electrodes were inserted bilaterally in the hemidiaphragms using the cervical incision thoraco-endoscopic approach (CITES) [6]. Diaphragm pacing (NeurRxDP4 stimulator, Synapse Biomedical Inc., Oberlin, OH, USA) was instituted within $2 \mathrm{~h}$ following the initiation of mechanical ventilation. Only one hemidiaphragm was stimulated throughout the experiments (right in two cases, left in one case), allowing comparison between mechanically ventilated hemidiaphragms (nonstimulated side) and mechanically ventilated with diaphragm pacing (MV+stim) ones (stimulated side). 30 min stimulation sessions were superimposed upon mechanical ventilation at $4 \mathrm{~h}$ intervals (18 breaths per minute, stimulation intensity $15 \mathrm{~mA}$, stimulation frequency $20 \mathrm{~Hz}$, inspiratory time $1.1 \mathrm{~s}$ and pulse width $150 \mu \mathrm{s}$, mechanical and electrical inspirations were synchronous). One animal suddenly died after $48 \mathrm{~h}$ of mechanical ventilation (\#3) and the two others (\#1 and \#2) were sacrificed after $72 \mathrm{~h}$ of mechanical ventilation. Costal diaphragm strips measuring $10 \times 8 \mathrm{~cm}$ were taken through an abdominal incision, immediately post mortem in animal \#3 and immediately before sacrifice in animals \#1 and \#2. Diaphragm samples were mounted on a small mound of $10 \%$ Gum Tragacanth placed on a cork disc and frozen in isopentane cooled with liquid nitrogen. Transverse serial cryosections ( $8 \mu \mathrm{m}$ thick) of the costal diaphragm were stained with haematoxylin and eosin, and analysed qualitatively for structural abnormalities. Other sections were stained for adenosine triphosphatase (ATPase pH 9.4) and fibre types were identified, according to their histochemical reactions, as slow twitch type I or fast-twitch type II fibres using ImageJ software (US National Institutes of Health, Bethesda, MD, USA). For each fibre type, an average cross-sectional area was determined from at least 150 fibres taken from six different fields in each hemidiaphragm, using NIS software (Nikon Instruments Europe B.V., Amsterdam, The Netherlands). Graphpad Prism (Graphpad Software, San Diego, CA) was used to calculate and plot the means and standard errors of the mean of measured quantities. Differences between the mechanically ventilated and $\mathrm{MV}+$ stim conditions were assessed using paired t-tests and were considered significant at $\mathrm{p}<0.05$.

In the three animals studied, mechanically ventilated hemidiaphragms showed signs of severe damage consisting of hypercontracted fibres with apparent lipid droplet accumulation and intense oedematous infiltrate of the interstitium leading to fibre disorganisation (fig. 1a). These structural abnormalities were not observed in the MV+stim hemidiaphragms, even after $72 \mathrm{~h}$ of mechanical ventilation (fig. 1a). The 
relative proportion of fibre types was not different between the mechanically ventilated and the MV+stim hemidiaphragms. Signs of diaphragm fibre atrophy were present in both the mechanically ventilated and MV+stim hemidiaphragms (fig. 1b) after $72 \mathrm{~h}$ (note the smaller type 1 fibre area in the animals mechanically ventilated for $72 \mathrm{~h}$ compared to the $48 \mathrm{~h}$ one, fig. 1c, middle), but these signs were markedly less pronounced in all the MV+stim hemidiaphragms. The surface area of type 2 fibres, the main fibre type in the sheep diaphragm [7], was significantly greater at $72 \mathrm{~h}$ in the MV+stim hemidiaphragms (fig. 1c, bottom) than in the mechanically ventilated hemidiaphragms. The area of type 2 fibres was smaller in the animals mechanically ventilated for $72 \mathrm{~h}$ than in the $48 \mathrm{~h}$ one on the mechanically ventilated side, but not on the MV+stim side.

This is a preliminary study, involving very few animals and a limited analysis of diaphragm structure, biology and function. In particular, we did not measure diaphragm contractility. Its preservation would have been expected in the paced hemidiaphragms, but this will have to be specifically demonstrated because
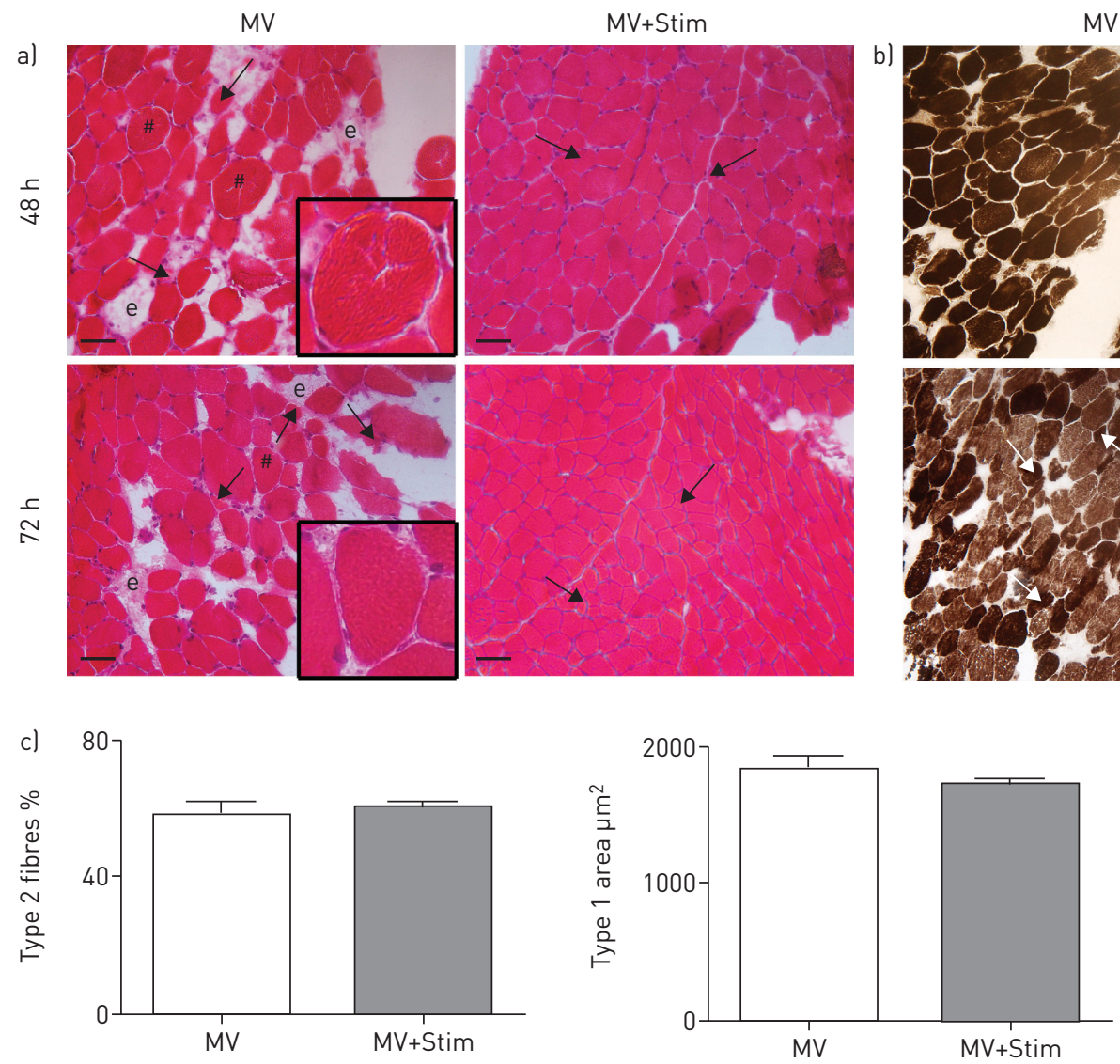

MV
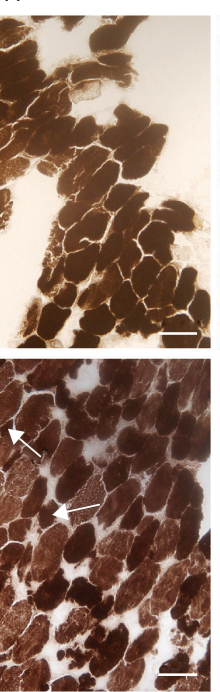
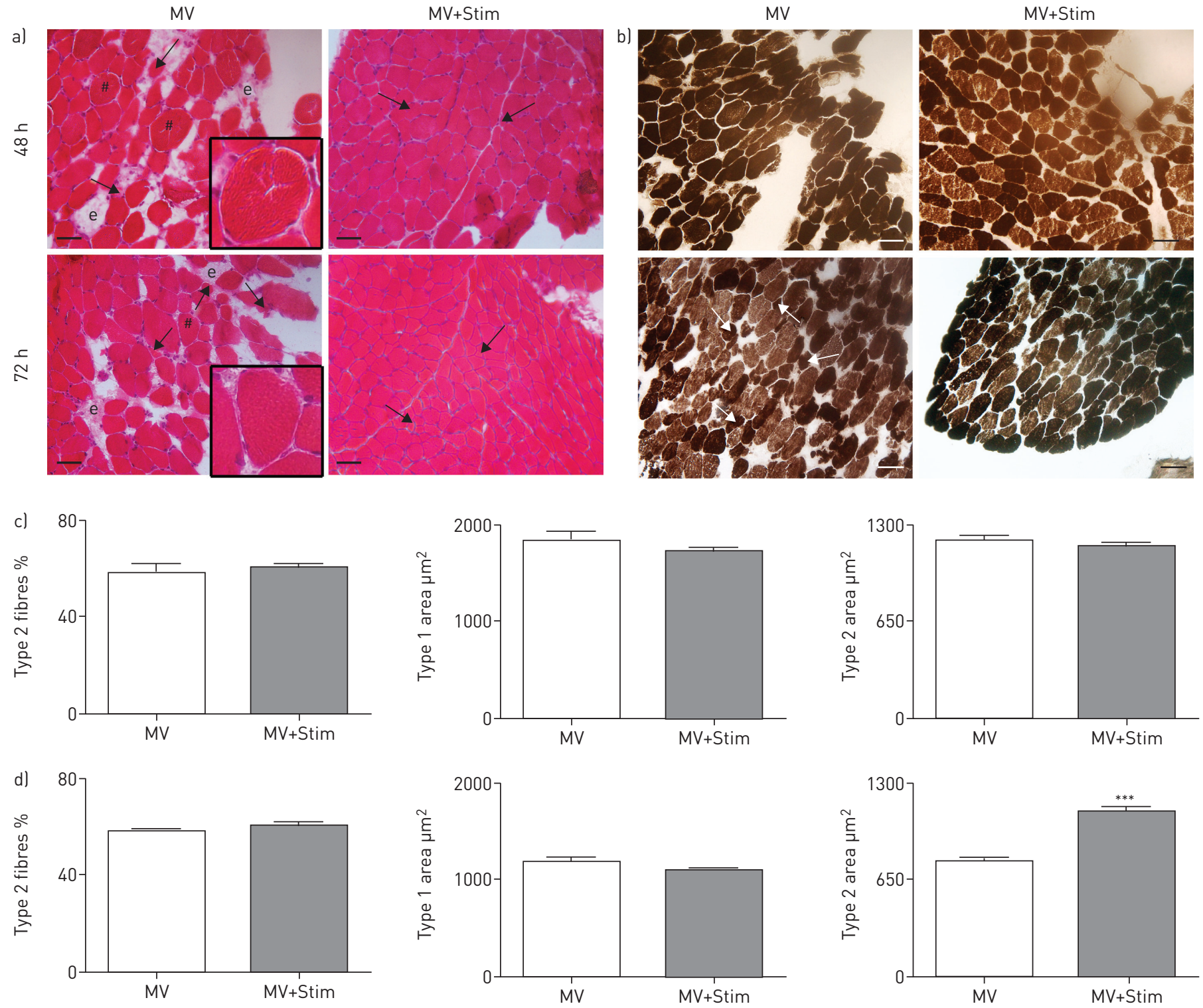

FIGURE 1 Morphological comparison between the hemidiaphragms on the mechanical ventilation only side (MV) and the hemidiaphragms on the mechanical ventilation plus diaphragm pacing side (MV+stim). a) Haematoxylin and eosin staining reveals hypercontracted fibres (\#) and interstitial oedema (e) in the MV diaphragms, both in the animal ventilated for $48 \mathrm{~h}$ and in the animals ventilated for $72 \mathrm{~h}$; arrows indicate atrophic fibres. Insert: close up view showing lipid droplets accumulation within hypercontracted fibres. Scale bar $=50 \mu \mathrm{m}$. b) ATPase staining at pH 9.4, fibres were identified as type 1 or type 2 (darker) according to their histochemical reactions; arrows indicate atrophic fibres. Scale bar $=50 \mu \mathrm{m}$. Proportion of type 2 fibres as a percentage of the total fibres (left), type 1 fibre area in $\mu \mathrm{m}^{2}$ (middle) and type 2 fibre area in $\mu \mathrm{m}^{2}$ (right) in c) the animal ventilated for $48 \mathrm{~h}$ and d) the two animals ventilated for $72 \mathrm{~h}$. ${ }^{* * *}: \mathrm{p}<0.001$ versus nonpaced diaphragm. 
the preservation of muscle structure is not necessarily associated with the preservation of muscle strength. Therefore, our results do not go beyond a proof of concept and cannot be considered conclusive in any manner. Importantly, our data pertain to healthy animals, as do all the currently available data about experimental VIDD. This calls for caution regarding extrapolation to acutely ill humans. Our observations are, however, worth reporting at this very early stage because they seem to provide the first experimental arguments supporting a putative utility of diaphragm pacing to prevent VIDD. Indeed, in our animals, the paced hemidiaphragms exhibited strikingly less damage (fig. 1a) and less type 2 fibre atrophy (fig. 1b-d) than the nonpaced ones. The design of the protocol (a large animal study conducted in intensive care unit (ICU)-like conditions; comparison of paced hemidiaphragms with non-paced ones in the same animals) lends strength to our observations. Muscle damage, as depicted in figure 1a, has not specifically been described as a feature of VIDD before, but these observations are consistent with those made in human VIDD using electron microscopy [8]. The presence of lipid droplets within the hypercontracted muscle fibres is also in line with the metabolic muscle dysfunction that has been described in human VIDD [4]. Finally, type 2 fibre atrophy is also concordant with the available VIDD data, in both animals and humans [2]. None of these features were present in the MV+stim hemidiaphragms in our animals. Diaphragm pacing could be protective against VIDD by opposing several of its mechanisms. It could counteract a "disuse atrophy" component and preserve muscle mass, in a manner similar to what has been described with electrostimulation of limb muscles in mechanically ventilated patients [9]. It could exert an antiinflammatory effect, as described for other types of electrical muscle stimulation [10]. It could also prevent eccentric or strain injuries provoked by the ventilation-induced stretching of a tonic diaphragm. All of the above hypotheses will have to be tested. Minimally invasive techniques for temporary diaphragm pacing in humans are being developed, including transcutaneous repetitive magnetic stimulation [11] and transvenous electrical stimulation $[12,13]$. Therapeutic phrenic nerve stimulation in critically ill patients is, therefore, within the realm of possibility in the near future, for the prevention or treatment of VIDD or other ICU-related diaphragm disorders. We submit that our data, as rudimentary as they may be, provide a basis for future studies of this approach. Our observations will have to be corroborated and consolidated in much larger animal groups, with a detailed description of the biological and physiological effects of diaphragm pacing during mechanical ventilation. Many questions will have to be answered: to identify optimal stimulation protocols; to describe the long-term effects, keeping in mind that low-frequency diaphragm stimulation tends to promote the development of type 1 fibres [14]; to identify deleterious effects due to electrical stimulation itself [15] or to clinical contexts such as sepsis where muscle stimulation could be counterproductive; to determine the best target population (will diaphragm pacing be restricted to patients in controlled ventilatory modes because of coma or any other reason? How does it compare with the maintenance of some spontaneous breathing activity when possible?); and, finally, to establish a riskbenefit balance.

0 @ERSpublications

In animals, implanted phrenic stimulation attenuates diaphragm type 2 fibre atrophy after $72 \mathrm{~h}$ of mechanical ventilation http://ow.ly/kTzHT

Hicham Masmoudi ${ }^{1,2}$, Catherine Coirault ${ }^{3,4}$, Alexandre Demoule ${ }^{3,5}$, Julien Mayaux ${ }^{5}$, Maud Beuvin ${ }^{3,4}$, Norma Romero ${ }^{3,4}$, Jalal Assouad ${ }^{2,6}$ and Thomas Similowski ${ }^{5,6}$

${ }^{1}$ Inserm U633, Paris, ${ }^{2}$ Dept of Thoracic Surgery, Assistance Publique-Hôpitaux de Paris APHP, Tenon Hospital, Paris, ${ }^{3}$ Inserm UMR_S974, CNRS UMR 7215, UPMC-University Paris 6 UM76, Pitié-Salpêtrière Hospital, Paris, ${ }^{4}$ Institute of Myology, Paris, ${ }^{5}$ Dept of Respiratory and Intensive Care Medicine, Assistance Publique-Hôpitaux de Paris APHP, PitiéSalpêtrière Hospital, Paris, and ${ }^{6}$ Paris 6 University, ER10upmc, Paris, France.

Correspondence: T. Similowski, Service de Pneumologie et de Réanimation Médicale, Groupe Hospitalier Pitié-Salpêtrière Charles Foix - Division Montyon, 47-83 Bd de l'Hôpital, 75651 Paris Cedex 13, France. E-mail: thomas.similowski@ psl.aphp.fr

Received: Mar 132013 | Accepted after revision: Mar 252013

Support statement: Synapse Biomedical Inc. provided the stimulation electrodes free of charge for this study and lent the phrenic nerve stimulators.

Conflict of interest: Disclosures can be found alongside the online version of this article at www.erj.ersjournals.com

Acknowledgements: The authors gratefully thank Julie Piquet (Laboratoire de recherches Biochirurgicales, Fondation Alain Carpentier Centre de Recherche Cardiovasculaire, Assistance Publique-Hôpitaux de Paris APHP, Hôpital Européen Georges Pompidou, Paris, France), Jérôme Cecchini, Julie Delemazure and Romain Persichini (all Dept of Respiratory and Intensive Care Medicine, Assistance Publique-Hôpitaux de Paris APHP, Pitié-Salpêtrière Hospital, Paris, France) for their involvement in the experiments and their support in animal management and intensive care. They thank Moustapha Diop, Synapse Biomedical Europe Inc, for friendly help and technical support. They thank Synapse Biomedical Inc., for lending the phrenic nerve stimulator and providing the stimulation electrodes free of charge. They also thank Philippe 
Menasche, head of the Laboratoire de recherches Biochirurgicales Fondation Alain Carpentier (Centre de Recherche Cardiovasculaire, Assistance Publique Hôpitaux de Paris; unité INSERM U633, Paris, France) for welcoming the experiments in his experimental facilities.

For editorial comments see page 12.

\section{References}

1 Vassilakopoulos T, Petrof BJ. Ventilator-induced diaphragmatic dysfunction. Am J Respir Crit Care Med 2004; 169: 336-341.

2 Jaber S, Jung B, Matecki S, et al. Clinical review: ventilator-induced diaphragmatic dysfunction-human studies confirm animal model findings!. Crit Care 2011; 15: 206.

3 Levine S, Nguyen T, Taylor N, et al. Rapid disuse atrophy of diaphragm fibers in mechanically ventilated humans. N Engl J Med 2008; 358: 1327-1335.

4 Picard M, Jung B, Liang F, et al. Mitochondrial dysfunction and lipid accumulation in the human diaphragm during mechanical ventilation. Am J Respir Crit Care Med 2012; 186: 1140-1149.

5 Pavlovic D, Wendt M. Diaphragm pacing during prolonged mechanical ventilation of the lungs could prevent from respiratory muscle fatigue. Med Hypotheses 2003; 60: 398-403.

6 Assouad J, Masmoudi H, Steltzlen C, et al. Minimally invasive trans-mediastinal endoscopic approach to insert phrenic stimulation electrodes in the human diaphragm: a preliminary description in cadavers. Eur J Cardiothorac Surg 2011; 40: e142-e145.

7 Finkelstein DI, Andrianakis P, Luff AR, et al. Developmental changes in hindlimb muscles and diaphragm of sheep. Am J Physiol 1992; 263: R900-R908.

8 Jaber S, Petrof BJ, Jung B, et al. Rapidly progressive diaphragmatic weakness and injury during mechanical ventilation in humans. Am J Respir Crit Care Med 2011; 183: 364-371.

9 Gerovasili V, Stefanidis K, Vitzilaios K, et al. Electrical muscle stimulation preserves the muscle mass of critically ill patients: a randomized study. Crit Care 2009; 13: R161.

10 Lambernd S, Taube A, Schober A, et al. Contractile activity of human skeletal muscle cells prevents insulin resistance by inhibiting pro-inflammatory signalling pathways. Diabetologia 2012; 55: 1128-1139.

11 Adler D, Gottfried SB, Bautin N, et al. Repetitive magnetic stimulation of the phrenic nerves for diaphragm conditioning: a normative study of feasibility and optimal settings. Appl Physiol Nutr Metab 2011; 36: 1001-1008.

12 Hoffer JA, Tran B, Tang J, et al. Diaphragm pacing with intravenous electrodes. 15th Annual Conference of the International Functional Electrical Stimulation Society, Vienna 2010; 012.

13 Ponikowski P, Javaheri S, Michalkiewicz D, et al. Transvenous phrenic nerve stimulation for the treatment of central sleep apnoea in heart failure. Eur Heart J 2012; 33: 889-894.

14 Marzocchi M, Brouillette RT, Klemka-Walden LM, et al. Effects of continuous low-frequency pacing on immature canine diaphragm. J Appl Physiol 1990; 69: 892-898.

15 Nosaka K, Aldayel A, Jubeau M, et al. Muscle damage induced by electrical stimulation. Eur J Appl Physiol 2011; 111: $2427-2437$.

\section{Quality of life after lung resection is not associated with functional objective measures}

\section{To the Editor:}

Patient and physician perspectives about surgical risk may differ. Physicians are mostly focused on objective end-points (i.e. mortality and survival), whereas most patients are worried about permanent physical and emotional disability resulting from the operation [1]. Can objective clinical information be used to predict patient-reported health status?

In the attempt to respond to this question, we studied 221 consecutive patients submitted to major anatomic pulmonary resections (204 lobectomy and 17 pneumonectomy) during a 36-month period. All patients had a pre-operative measurement of maximum oxygen uptake $\left(V^{\prime} \mathrm{O}_{2} \max \right)$, as a part of their routine pre-operative functional work-up, and a complete assessment of their pre-operative and post-operative ( 3 months after surgery) quality of life. All patients gave their consent for inclusion of their clinical data in our institutional database for clinical and scientific purposes and the Institutional Review Board of our hospital approved the study. No formal pre-admission or post-discharge physiotherapy or psychological support programmes were administered in this series. Neurological or psychotropic personal medications, if present, were generally resumed the day after surgery. 